\title{
CAMBIO CLIMÁTICO Y MIGRACIONES LABORALES EN LA FRONTERA SUR DE MÉXICO
}

\author{
LAURA ELENA RUIZ MEZA ${ }^{1}$ \\ Recibido el 20 de marzo de 2012 y aprobado el 9 de \\ septiembre de 2012
}

\section{RESUMEN}

A la pobreza persistente en México, se suman las manifestaciones del cambio climático, cuyos impactos más significativos se observan en el sector agrícola y en la soberanía alimentaria. En la entidad fronteriza de Chiapas, con los índices más bajos de desarrollo humano, los estragos ocasionados por los eventos climáticos extremos de los últimos años agravaron las condiciones de vulnerabilidad de la población e incrementaron significativamente los flujos migratorios. El cambio climático tiene serios efectos en el empleo y en los mercados de trabajo del sector rural, el más afectado. En el contexto de los efectos provocados por la variabilidad climática y la crisis del sector agropecuario, la dinámica migratoria ha adquirido mayor complejidad en los últimos años. Se muestra que las migraciones internacionales han tenido como destino las entidades fronterizas del sur de México, pero cada vez más figuran como regiones de tránsito y origen de los flujos migratorios hacia los Estados Unidos de Norteamérica, fenómenos interrelacionados que tienen en común el aumento de la pobreza por efecto del cambio climático.

\section{PALABRAS CLAVE:}

Cambio climático, migración, vulnerabilidad social

\section{CLIMATE CHANGE AND LABOR MIGRATION IN THE MEXICAN SOUTHERN BORDER}

\begin{abstract}
To the persistent poverty conditions existing in Mexico, manifestations of climate change, whose most significant impact is observed in the agricultural sector and in food sovereignty, are added. In Chiapas, southern Mexico, with the lowest human development index, the disasters caused by extreme weather events in recent years made worse the vulnerability of the population and significantly increased migration flows. Climate change has serious effects on employment and labor markets in the rural sector which is the most affected sector. In the context of the effects
\end{abstract}


caused by climate variability and the crisis in the agricultural sector, the dynamics of migration has become more complex in recent years. This paper shows that international migration has had the border states of southern Mexico as destination, but increasingly they appear as transit regions and origin of migration to the United States of America, interrelated phenomena that have in common increased poverty because of climate change

\section{KEYWORDS:}

Climate change, migration, social vulnerability

\section{INTRODUCCIÓN}

En México, las manifestaciones del cambio climático se suman a las condiciones estructurales de pobreza. Según datos oficiales, el $46.2 \%$ de la población vive en situación de pobreza y otro $10.4 \%$ en pobreza extrema; en el contexto de la crisis económica se ha incrementado la población que carece de acceso a la alimentación y reducido el ingreso real de los hogares; el $80 \%$ de los mexicanos de zonas rurales se clasifica como pobre (CONEVAL, 2012). Tales niveles de inequidad social, junto a la situación geográfica del país y el severo deterioro ambiental generan condiciones de aguda vulnerabilidad social a los eventos hidrome $\neg$ teorológicos extremos, que se han presentado con mayor frecuencia e intensidad en los últimos años. Mientras que la desertificación avanza en el norte y centro del país, el sur se ve impactado por tormentas tropicales, huracanes e inundaciones. Durante la década de 1990 más del $90 \%$ de las personas que fallecieron por los efectos de fenómenos naturales perdieron la vida en huracanes, sequías, tormentas e inundaciones; alrededor de 20 millones de personas habitan áreas susceptibles al impacto de huracanes (Landa, et al. 2008) ${ }^{2}$.

Los impactos más significativos del cambio climático se observan en el sector agrícola, con serios efectos en el empleo y la seguridad alimentaria, pues se trata de un sector particularmente sensible a los cambios graduales en la temperatura y la precipitación (INE-SEMARNAT, 2006; Fetzek, 2009). En el país, una cuarta parte de la población subsiste de actividades relacionadas con la agricultura, y la gran mayoría vive en condiciones de pobreza (Sánchez-Cohen, et al. 2008). Dada su mayor frecuencia en las últimas décadas, los desastres 
relacionados con los cambios en el clima en el período 1980-2005 afectaron principalmente al sector agrícola y fueron responsables del $80 \%$ de las pérdidas económicas generadas por desastres naturales (Saldaña \& Sandberg, 2009). Además, la tierra cultivada asegurada en México disminuyó de $40 \%$ a $10 \%$ entre 1990 y 2000, período en el que los daños a los cultivos causados por los eventos climáticos extremos se duplicó (Saldaña \& Sandberg, 2009).

A partir de la década de 1980, los efectos sociales de las medidas de ajuste estructural y de libre comercio -junto a las pérdidas económicas generadas por las amenazas naturales y la exigua respuesta gubernamental para reducir los efectos nocivos de ambos procesos- han debilitado las condiciones de trabajo y de empleo, causando situaciones de empobrecimiento que han generado un aumento dramático en la migración durante las últimas décadas (SAPRIN, 2002). De 1990 a 2005 la población residente en Estados Unidos de origen mexicano aumentó de 4.4 millones a 11.8 millones de personas. Actualmente, en Estados Unidos viven más de 30 millones de personas de origen mexicano y representan el grupo más numeroso de inmigrantes en los Estados Unidos (CONAPO, 2007).

El debate sobre los efectos del cambio climático y la movilidad de las personas está ganando cada vez más interés científico y político a nivel internacional (IPCC, 2001; IOM, 2008; Couldrey \& Herson, 2008). Desde la década del 90, el Grupo Intergubernamental de Expertos sobre el Cambio Climático señalaba a la migración como una de las más graves consecuencias de dicho cambio, pues millones de personas tendrían que desplazarse a causa de la erosión de la línea costera, las inundaciones y los estragos en la agricultura. En varias regiones del mundo se detectan procesos migratorios como respuesta de adaptación a la tensión debida al clima, y se sugiere el término "migrante forzoso por motivos climáticos" para dar cuenta del fenómeno del desplazamiento no voluntario de la población a causa de los efectos del cambio en el clima (Brown, 2008).

Varios estudios recientes, realizados alrededor del mundo, han abordado los impactos de las variaciones climáticas en la migración y, aunque se reconoce que no se puede determinar una relación única entre cambio climático antropogénico y migración, pues intervienen diversos factores sociales, económicos y ambientales en los flujos migratorios, sí se ha demostrado que la mayor frecuencia y magnitud de los fenómenos climáticos extremos y de degradación ambiental actúan como un acelerador del 
proceso migratorio, obligando a la población a buscar lugares que les puedan ofrecer medios de vida más estables y seguros (Alscher, 2008-2009; EACH-FOR, 2009; CARE-UNU, 2009; Kartiki, 2011).

En las entidades fronterizas del sur del México, que presentan bajos índices de desarrollo humano, los estragos ocasionados por los eventos climáticos extremos de los últimos años han agravado las condiciones de vulnerabilidad de la población e incrementado significativamente los flujos migratorios. En este artículo se presenta una perspectiva de dicho fenómeno en el contexto de los efectos provocados por la variabilidad climática y por la crisis del sector agropecuario, dando cuenta de la complejidad que ha adquirido la dinámica migratoria en los últimos años.

Cambio climático y migración en México. Estudios recientes realizados en México y en el estado sureño de Chiapas demuestran que los desastres naturales recurrentes, en su mayoría relacionados con el clima, junto con la disminución de los ingresos familiares y la falta de acceso a medios económicos que permitan la recuperación de los activos perdidos en las catástrofes, son factores estimulantes de la migración (Saldaña \& Sandberg, 2009; Alscher, 2008-2009; Jungehülsing, 20102011; Ruiz, 2011). La migración es considerada como una estrategia adoptada por los hogares afectados para afrontar o responder a los impactos dañinos de los eventos climáticos extremos en sus medios de vida, y empieza a cobrar mayor importancia en aquellos sectores y grupos de la población con mayores grados de vulnerabilidad. La migración a largo plazo o permanente, a diferencia de la migración estacional o temporal, es cada vez más común, particularmente entre las generaciones más jóvenes.

En las poblaciones rurales afectadas por fenómenos asociados a los cambios en los patrones de precipitación y temperatura, la sustitución de cultivos u otras opciones de mejoramiento económico pocas veces se exploran como opciones, más bien se tiende a hacer uso de la emigración (Saldaña \& Sandberg, 2009). Ello es particularmente evidente ante la ausencia de políticas públicas de reactivación del empleo y de fomento a la producción agrícola de subsistencia que permita a los pequeños agricultores fortalecer su producción de alimentos y aumentar sus ingresos con el cambio a cultivos más rentables ${ }^{4}$.

En un contexto de profundización de la crisis del sector agropecuario, de aumentos en la frecuencia e intensidad en eventos climáticos extremos y de ausencia de 
instrumentos económicos para la cobertura contra los riesgos de desastre, el amplio sector de los agricultores de subsistencia no está en condiciones de recuperarse de su situación estructural de marginación, permaneciendo atrapados en un círculo vicioso que aumenta su vulnerabilidad. Dado que sus expectativas de incremento de ingresos no son optimistas en el contexto adverso en el que viven, la migración a las ciudades es la opción más viable que tienen para cubrir sus expectativas de ingresos futuros. Si las dimensiones de los impactos ambientales y económicos exceden la capacidad de la población vulnerable para responder y adaptarse en sus lugares de origen, la migración a regiones que ofrezcan mayores ingresos puede ser una estrategia viable, incluso ante las actuales restricciones a la movilidad laboral y las dificultades para cruzar las fronteras internacionales.

El estudio realizado por Saldaña y Sandberg (2009) en 2,443 municipios de México, revela que las regiones del país más afectadas por los desastres naturales durante la década de 1990 han experimentado los más altos índices de migración, las cuales además presentan altos niveles de pobreza y disminución de los ingresos por la caída en los precios de los cultivos. Asimismo, los autores afirman que los municipios que han experimentado una mayor reducción de los salarios son más proclives a la emigración, y demuestran que la frecuencia de catástrofes climáticas estimula a las personas a emigrar a las grandes ciudades, debido a que en los municipios pequeños con actividades económicas poco dinámicas los trabajadores suelen no encontrar empleo. La limitada capacidad de la economía rural para proporcionar empleo ha aumentado significativamente el flujo de la migración a las grandes ciudades y al extranjero. Los autores concluyen que aquellos municipios más propensos a la emigración en México presentan un conjunto de características comunes: situación de marginación, disminución sustancial de los ingresos, subsistencia basada en la agricultura de granos básicos, afectaciones por los frecuentes desastres naturales y lejanía de los grandes centros urbanos, entre las más importantes.

Se ha mencionado de manera reiterada que la población empobrecida es la más vulnerable a los impactos del cambio climático, pues la carencia de recursos limita su capacidad de respuesta y adaptación (CARE, 2009). Dado que la insuficiencia de ingreso ya no es el único criterio para definir la pobreza, pues importa igualmente la disponibilidad de recursos o activos de distinto tipo: natural, físico, financiero, humano y social, la habilidad de los hogares para ensayar diferentes estrategias de vida depende de los activos que posean para alcanzar su 
bienestar (Ellis, 2000); cuando éstos son afectados y reducidos por los desastres naturales, la vulnerabilidad de los hogares se incrementa.

La vulnerabilidad es un concepto fundamental en el análisis de las capacidades sociales de respuesta ante el cambio climático. Ésta se concibe como el estado de susceptibilidad a los daños causados por la exposición a tensiones asociadas a cambios ambientales y sociales, así como a la ausencia de capacidad para recuperarse de tales impactos (Adger, 2006). Mientras que la capacidad de "ajuste" (Lavell, et al. 2012) se refiere a los procesos de respuesta y reacción a los impactos de las amenazas vinculados a la necesidad de sobrevivir, la capacidad de adaptación implica ajustes a las cambiantes condiciones del entorno, así como procesos de transformación y reorganización para mantener el nivel de vida, por lo tanto, requiere de acción planificada, con una perspectiva de mediano y largo plazo (Birkmann, 2009). Ante el incremento de la vulnerabilidad social conviene preguntarse si la creciente migración que se experimenta en la frontera sur de México podría considerarse como una medida de ajuste para afrontar el impacto de las amenazas naturales, o empieza a constituirse en un proceso adaptativo de largo aliento.

La migración internacional en la frontera sur. La frontera sur de México ${ }^{5}$ es escenario de un conjunto de fenómenos asociados a la migración internacional que ha adquirido relevancia social, económica y política por ser a la vez destino, tránsito y origen de migrantes (Ángeles, 2009). La migración transnacional ha experimentado un rápido crecimiento en la última década y avanza a pasos más rápidos que en otras regiones de México con mayor tradición migratoria. Entidades como Chiapas, Veracruz, Tabasco, Campeche, Quinta Roo y Yucatán, antes ajenos al fenómeno migratorio, ahora son entidades en las que el proceso se consolida como la principal estrategia de vida de amplios sectores de la población (Serrano \& Tuñón, 2009).

Si bien la frontera sur de México había sido escenario tradicional de migraciones laborales que llegaban provenientes de Guatemala, desde finales del siglo XX se ha generado un incremento significativo de migrantes centroamericanos que intentan cruzar el territorio mexicano para llegar a la frontera con Estados Unidos e ingresar a tal país, a los que se han sumando migrantes que desde Chiapas persiguen el mismo propósito, configurando un contexto de mayor complejidad en la migración (Ángeles, 2009). 
El origen de los movimientos poblacionales en la frontera de México-Guatemala data desde finales del siglo XIX, cuando los trabajadores agrícolas originarios de Guatemala desempeñaron un papel clave en el desarrollo económico de la región al aportar mano de obra en las fincas cafetaleras $y$, después, en las plantaciones de algodón, cacao y plátano durante el siglo XX. En los últimos años se han diversificado los tipos de trabajo donde se emplean, pues además de las actividades agrícolas y el trabajo doméstico, también laboran en trabajos más urbanos relacionados con el sector comercio y servicios; incluso se vinculan con el comercio sexual y la venta de estupefacientes. Se trata de empleos temporales y precarios, totalmente marginales. Según la encuesta de Migración en la Frontera Guatemala-México realizada en el año 2004 , el $82 \%$ de los migrantes permaneció hasta un mes en su empleo, lo que sumado a su condición de indocumentados revela la inexistencia de condiciones mínimas que les asegure un empleo digno y decente (Ángeles, 2009).

Con la instrumentación de políticas neoliberales en México a partir de la década de 1980 (Calva, 2004), el agro se sumió en una profunda crisis que dio inicio a un proceso migratorio de grandes proporciones en algunas regiones y entidades del sur del país. A este flujo migratorio se sumaron trabajadores centroamericanos que ingresan por la frontera sur de México con la intensión de llegar a Estados Unidos de Norteamérica (Villafuerte \& García, 2006). Igualmente, uno de los impactos sociales más visibles del cambio climático es el incremento en los flujos migratorios, factor que se suma a la pobreza extrema en la que vive la población de la región mesoamericana. En las zonas afectadas por los fenómenos naturales, como los huracanes Mitch en 1998 y Stan en 2005, la emigración se incrementó sustancialmente, como se revisará más adelante. Los grupos de población migrante se caracterizan por su aguda vulnerabilidad y la violación sistemática de sus derechos humanos, incluso son víctimas del crimen organizado.

Pobreza rural y precariedad laboral. Chiapas es un estado predominantemente rural, con los más altos niveles de desigualdad y rezago social del país, lo que contrasta con su riqueza biológica y cultural. Datos oficiales indican que el $78.4 \%$ de la población vive en situación de pobreza y pobreza extrema, a la que se suma el $13.1 \%$ de la población en condición de vulnerabilidad por carencias sociales (CONEVAL, 2012). El $27.2 \%$ de sus habitantes es de origen indígena (INEGI, 2010). A la condición de pobreza estructural se agrega la exclusión social generada por la políticas neoliberales de ajuste que se tradujeron en 
una drástica reducción de los subsidios, de los programas de fomento e inversión pública y el desmantelamiento del aparato oficial; entre 1994 y 2000 la inversión del gobierno federal al campo cayó en un 90\% (Villafuerte \& García, 2007). La apertura comercial con el Tratado de Libre Comercio con América del Norte (TLCAN) y la crisis de los precios de los productos agrícolas, en especial del café, son otros factores que han tenido efectos devastadores para las economías campesinas, y en general para el sector agropecuario ${ }^{6}$.

En Chiapas, el modelo de agricultura de plantaciones y la ganadería extensiva se vio sensiblemente afectada por la apertura comercial y la desregulación económica. Se observa una pérdida acumulada de rentabilidad, lo que ha generado fuertes cambios en los mercados laborales y amplio desempleo. Villafuerte y García (2007) señalan que la falta de opciones de empleo fuera del sector agropecuario mantiene a una fuerza laboral muy grande vinculada a las actividades del sector primario, pero en condiciones de elevado subempleo, lo que ha originado un desplazamiento de la población a los mercados laborales del norte de México y Estados Unidos. Mediante un análisis de los datos censales, en los siguientes renglones se muestran las dimensiones de la pobreza y desempleo rural en Chiapas, que han mostrando un incremento significativo en las últimas décadas.

La Población Económicamente Activa (PEA) agropecuaria ha experimentado una disminución relativa al pasar de $57 \%$ en 1980 , a $47 \%$ en 2000 y a $37.3 \%$ en 2009 . Frente a la ausencia de un importante sector industrial en Chiapas, la PEA en el sector comercio y servicios ha crecido de manera extraordinaria al pasar de $13 \%$ en 1980 , a $37 \%$ en 2000 y a 48\% en 2009 (Villafuerte \& García, 2007; INEGI, 2009). En las regiones de la entidad que fueron más afectadas por el huracán Stan en 2005: Sierra, Soconusco e Istmo-Costa, la PEA ocupada en las actividades agropecuarias continúa siendo destacada, pues supera el $50 \%$, y en algunos municipios es mayor a $80 \%$, sobre todo en la Región Sierra que presenta índices de muy alta marginación (INEGI, 2005).

En 1980, el sector agropecuario aportaba el $28.6 \%$ del producto interno bruto (PIB) estatal, mientras que el sector comercio y servicios contribuía con el $47.2 \%$. En el año 2000 la contribución del sector agropecuario al PIB se desplomó al $15.6 \%$, mientras que la del comercio y servicios se elevó a $63.4 \%$ (Villafuerte \& García, 2007). En 2008 , el aporte del sector agropecuario bajó al $8.5 \%$, y el del sector comercio y servicios ascendió al $66.3 \%$ del PIB estatal (INEGI, 2009). Otro de los efectos de las políticas 
neoliberales en Chiapas ha sido la estrechez de la estructura productiva, pues se basa sólo en tres cultivos comerciales: café, banano y caña de azúcar, en los que se centra la dinámica económica del agro.

Respecto al empleo, del total de población ocupada en el sector agropecuario en 1990 , el $63.5 \%$ fue clasificada como trabajadores por cuenta propia, $21.9 \%$ fueron empleados y jornaleros, y menos del $1 \%$ fueron considerados como patrones o empresarios. En el año 2000 , bajó a $57 \%$ la proporción de trabajadores por cuenta propia, $24.6 \%$ fueron empleados y jornaleros y $0.8 \%$ empresarios. Cabe destacar que el trabajador familiar no remunerado se incrementó de $8.8 \%$ en 1990 a $13.2 \%$ en 2000 , cifras que revelan un proceso de empobrecimiento (Villafuerte \& García, 2007).

En las últimas décadas, la tendencia a la pauperización del agro chiapaneco se puede observar a través del indicador de ingresos de la población vinculada a las labores agropecuarias: en 1980 , el $48.5 \%$ de la PEA no recibió ingresos; y de los que declararon ingresos, el $19.7 \%$ obtuvo menos de un salario mínimo y $25.4 \%$ entre uno y dos salarios mínimos ${ }^{7}$. En 1990, luego de una década de ajuste estructural, estas cifras se elevan notablemente. De la PEA ocupada en el sector rural que declaró haber obtenido ingresos, alrededor del $70 \%$ percibió menos de un salario mínimo y el $16.2 \%$ obtuvo entre uno y dos salarios mínimos; así que $86.2 \%$ de la PEA que declaró ingresos se encontraba en pobreza y pobreza extrema. En el año 2000, tal grupo había crecido al $90.7 \%$ de la población laboral en el campo, indicadores del severo proceso de pauperización de la gran mayoría de la población rural en Chiapas (Villafuerte \& García, 2007).

A estos datos se agrega el elevado subempleo. Según el censo agrícola de 1990 , el $79.2 \%$ de la fuerza laboral empleada en las unidades de producción no obtuvo remuneración; y el $83.3 \%$ de la mano de obra remunerada era eventual. La mano de obra desempleada y subempleada contribuiría a engrosar los flujos migratorios hacia las ciudades, el norte del país y Estados Unidos en busca de su sobrevivencia. Recientemente, el Censo Agropecuario reveló que el $91.6 \%$ de los empleados en las actividades agropecuarias laboró menos de seis meses (INEGI, 2008) lo que indica que el subempleo se elevó y continuaba siendo de enormes proporciones.

Eventos climáticos extremos y procesos migratorios. En Chiapas, el cambio climático se ha manifestado en los últimos años con perturbaciones significativas en el régimen hidrológico, el aumento de los períodos de sequía 
y la incidencia de incendios forestales (Arellano, 1999). De acuerdo con un estudio sobre economía del cambio climático en México, Chiapas es la segunda entidad con menor capacidad de sobreponerse a los impactos del de tal cambio (Ibarrarán \& Rodríguez, 2007). Se estima que al menos el $75 \%$ de su territorio se verá afectado por sequías e inundaciones, lo que implica la afectación de la producción de granos básicos (Aguilar, 2006). Chiapas es el cuarto estado productor de maíz a nivel nacional y cuenta con una gran riqueza genética del grano, la cual corre el riesgo de verse afectada, además, con la reciente autorización de siembras experimentales de maíz transgénico (Enciso, 2012).

Han sido frecuentes los eventos meteorológicos que han impactado a Chiapas; desde 1985 se han presentado prácticamente todos los años (Ruiz, 2011). Por las dimensiones de sus daños, se hará mención de dos de ellos: las depresiones tropicales de 1998 y el huracán Stan de 2005. En 1998, Chiapas sufrió los estragos de los incendios forestales durante la prolongada temporada seca de ese año, que afectó una superficie de 198,808 hectáreas. Meses después, las intensas lluvias provocaron derrumbes e inundaciones severas, cuyos impactos se tradujeron en enormes pérdidas humanas y económicas. Se destruyeron 400 mil hectáreas de cultivos, $500 \mathrm{mil}$ pobladores perdieron su hogar y 400 personas fallecieron. La población rural resultó ser la más afectada (Valladares, et al. 1999).

Por su parte, el huracán Stan, en el año 2005, causó severos daños en 41 de los 118 municipios de Chiapas. Además de las pérdidas en vidas humanas, 25 mil familias sufrieron la destrucción total de sus viviendas; se colapsaron 6 mil km de caminos y carreteras, además de 200 puentes (Secretaría de Desarrollo Social, 2005). Se vieron afectadas 306,994 hectáreas en producción y 121,784 agricultores, así como las actividades pesqueras y forestales. El $75 \%$ de las tierras dedicadas al cultivo de café resultaron dañadas. En Guatemala, vastas regiones resultaron afectadas, áreas donde habita el $69 \%$ de la población y cuyo aporte al PIB es de $90 \%$ (Castellanos 2005, citado por Ángeles, 2009).

Los efectos de los eventos hidrometeorológicos extremos han agudizado aún más las precarias condiciones de vida de las poblaciones chiapanecas. Numerosos estudios sobre migración en México, Chiapas y Centroamérica coinciden en señalar que las amenazas climáticas han acelerado los ya existentes procesos migratorios, reconfigurando la actividad migratoria en la frontera sur de México (Ángeles \& Rojas, 2000, 2009; Villafuerte \& García, 
2006; Alscher, 2009). Así, en los últimos años, la migración en la frontera sur de México ha adquirido mayor dinamismo y complejidad, pues se ha convertido simultáneamente en lugar de destino, tránsito y origen de migrantes; en un territorio de inmigrantes, transmigrantes y emigrantes que conforman un mosaico complejo de migrantes rurales y urbanos (Ángeles \& Rojas, 2009; Villafuerte, 2008).

Como lugar de destino, en Chiapas se destacan las migraciones de trabajadores agrícolas procedentes de Guatemala. Los desequilibrios económicos entre campo y ciudad como los efectos de los huracanes han provocado que los servicios urbanos, el comercio y la industria de la construcción sean nuevas opciones de empleo para los trabajadores centroamericanos, incrementando su presencia en un mayor número de centros urbanos de las entidades de la frontera sur de México (Ángeles \& Rojas, 2009).

Chiapas también se ha convertido en lugar de tránsito, pues en la última década ha ocupado un lugar estratégico para las migraciones provenientes de Guatemala, Honduras y El Salvador, e incluso de otros países de América del Sur hacia los Estados Unidos (Villafuerte, 2008) $)^{8}$. La Encuesta sobre Migración de la Frontera Guatemala-México realizada en 2006 (INM, 2009), revela que los más afectados por el huracán Stan en 2005 fueron los trabajadores agrícolas, pues el $42.4 \%$ perdió su trabajo. La reactivación del mercado laboral favoreció a los trabajadores del sector de la construcción, no así a los que laboraban en el sector agropecuario, lo que muestra la dimensión del daño que sufrió tal sector. Los migrantes en tránsito hacia la frontera norte son víctimas de las extorsiones, asaltos y abusos por parte de las autoridades migratorias, de los delincuentes comunes y organizados. Estas violaciones a los derechos humanos de los migrantes van creando una cultura de abuso e impunidad en torno a la migración (Ángeles \& Rojas, 2009).

Como lugar de origen de la migración, Chiapas ha comenzado a figurar como una de las entidades con mayor dinámica migratoria hacia el norte del país y los Estados Unidos de Norteamérica. La emigración ha sido una estrategia usada por el campesinado para enfrentar los efectos adversos de los eventos climáticos en sus actividades productivas. Aunque el factor climático no es la única causa de la emigración rural, destaca en la intricada correlación de variables de orden político, social, económico y ambiental. Como lo afirman Villafuerte y García (2006), la explicación de la reciente dinámica migratoria en la entidad es compleja y no puede atribuirse 
exclusiva 7 mente a la crisis rural y a la pobreza, pues éstos son rasgos persistentes desde tiempo atrás sin que se hubiese establecido una relación directa con el fenómeno migratorio inter $\neg$ nacional.

Luego de los eventos climáticos de 1998, la industria ma $\urcorner$ quiladora establecida en el norte del país ofreció empleos a los damnificados, de manera que a fines de ese año 35 mil chiapanecos ya habían emigrado. Desde entonces, los negocios de transporte desde la frontera sur hacia los Estados Unidos han proliferado (Castro, 2005). Según la Encuesta sobre Migración en la Frontera Norte de México, los migrantes originarios de Chiapas deportados por la patrulla fronteriza se elevaron de 13,372 personas en 1999, a 30,523 en el año 2000 (Gobierno del Estado de Chiapas, 2004). En 2005, el 40\% de la población chiapaneca radicada en Estados Unidos eran originarios de las regiones más afectadas por los eventos climáticos extremos ya señalados (Olivera \& Sánchez, 2008).

El crecimiento exponencial de las remesas enviadas es otro indicador de la reciente emigración hacia Estados Unidos. El monto de las remesas se multiplicó 33 veces al pasar de 19.8 millones de dólares en 1995 a 655.3 millones en 2005 (Villafuerte \& García, 2007). En el año 2006 la cifra se elevó a 800 millones de dólares, equivalente al valor de la producción del sector primario, lo que sitúa a la entidad en el lugar número 11 a nivel nacional en el envío de remesas, por arriba de otras entidades de mayor tradición migratoria (Olivera \& Sánchez, 2008). En un contexto de profundización de la pobreza en el sector rural, las remesas empiezan a reemplazar los ingresos agrícolas. No obstante, la creciente importancia económica de las remesas no ha significado mayores oportunidades de inversión y empleo para las familias de los migrantes, ni ha implicado una reactivación de las economías campesinas; en el mejor de los casos contribuyen a mitigar la pobreza, contener el hambre y reducir el descontento social (Villafuerte, 2008) ${ }^{9}$.

Entre los impactos no deseados de la migración hacia Estados Unidos destaca el incremento de los hogares rurales encabezados por mujeres, quienes asumen la responsabilidad de garantizar la producción social de sus familias en condiciones adversas. Asimismo, tiene efectos en los mercados de trabajo, pues en los sectores productivos más dinámicos de Chiapas se generan empleos aún más precarios y marginales ocupados por trabajadores centroamericanos que carecen de oportunidades laborales en sus lugares de origen. 
Estos fenómenos han provocado la emergencia de flujos migratorios a los cuales se han incorporado nuevos migrantes que carecen de redes sociales de apoyo, entre los que se destacan las mujeres e infantes. Las mujeres proceden de diversos países centroamericanos para laborar como trabajadoras agrícolas, empleadas domésticas y en el sector comercio; ellas están expuestas de manera muy aguda a diversos riesgos que incrementan su vulnerabilidad (Rojas, 2007). Además de su condición de género, la discriminación que sufren se ve multiplicada por ser extranjeras indocumentadas, pobres e indígenas. $\mathrm{Se}$ incorporan en trabajos muy precarios, marginales, temporales $e$ inseguros que no son remunerados de manera justa. Algunas laboran temporalmente en Chiapas para obtener recursos que les permitan continuar su viaje hacia Estados Unidos, y fácilmente caen en redes de prostitución y tráfico de personas. En este grupo de migrantes temporales también se encuentran niños y niñas que suelen dedicarse a distintas actividades del sector servicios de manera informal. Los infantes corren el riesgo de ser explotados o abusados sexualmente (Ángeles \& Rojas, 2009). Por su parte, las mujeres migrantes originarias de Chiapas que aspiran a llegar a Estados Unidos se ven expuestas a altos riesgos por los numerosos obstáculos a vencer. Sus derechos humanos son violados al verse sometidas a asaltos, extorsión, violencia sexual, secuestro, maltratos, detención arbitraria, discriminación e incluso la muerte a manos de delincuentes y funcionarios corruptos.

Las migraciones que tienen como destino a Chiapas, pero también como lugar de tránsito y origen, son fenómenos interrelacionados que tienen en común el recrudecimiento de la pobreza y vulnerabilidad de la población por efecto del cambio climático. Los datos presentados dan cuenta de la complejidad que ha adquirido el fenómeno migratorio en los últimos años, así como la precariedad del empleo en los distintos sectores económicos. Tanto en Chiapas, como en Centroamérica, existe una incapacidad de la economía por generar los empleos adecuados y necesarios para cubrir las necesidades de la población creciente, ante ello las familias pobres han tenido que diversificar sus fuentes de subsistencia, siendo la migración una estrategia de respuesta de creciente importancia.

\section{REFLEXIONES FINALES}

- Los vínculos entre cambio climático, pobreza y migración en la frontera sur de México han sido explorados en el 
presente artículo, región caracterizada por ser ámbito de recepción y de tránsito de migrantes, pero también por ser zona de origen de flujos migratorios en años recientes, condición que da complejidad a la dinámica migratoria en la región. Se demostró que en un contexto de profundización de la crisis del sector agropecuario y de precariedad en el empleo, la migración se ha visto acrecentada por los nocivos efectos de los frecuentes e intensos eventos hidrometeorológicos extremos, lo que ha agudizado aún más las ya precarias condiciones de vida de la población. Las migraciones internacionales que tienen lugar a lo largo de la frontera sur son fenómenos interrelacionados que tienen en común la falta de opciones de vida, el recrudecimiento de la pobreza y la vulnerabilidad de la población por efecto del cambio climático.

- La degradación ambiental y los impactos del cambio climático agudizan la migración, pero no dejan de estar articulados con los factores de orden social, económico y político, factores que en conjunto evidencian las agudas desigualdades sociales en el acceso y control de los recursos clave para la subsistencia, y que configuran un contexto de mayor vulnerabilidad y riesgo para los medios de vida de la población.

- La migración se ha ensayado como un mecanismo de respuesta para afrontar los impactos de los eventos climáticos extremos; nuevos estudios permitirán demostrar si se trata de un mecanismo de adaptación para las poblaciones afectadas. La generación de nuevos conocimientos permitirá identificar los vínculos entre cambio climático, vulnerabilidad social y migración y hará visibles las capacidades locales que entran en juego en los procesos de adaptación. Ello podría facilitar la incorporación de la perspectiva de equidad social en las diferentes intervenciones en materia de gestión territorial y cambio climático, ausentes hasta ahora.

- Los movimientos ambientalistas en México están participando activamente en el debate nacional e internacional sobre cambio climático, y han puesto énfasis en la preservación ambiental, el uso de energías renovables y la generación de empleos verdes. Sin embargo, los movimientos sindicalistas aún no figuran en el debate, pese a encontrase notablemente afectados por las políticas de privatización impulsadas en el sector rural y en el sector energético. El compromiso y papel de los movimientos sindicales puede orientarse para demandar la creación de empleos dignos, de tal manera que la emigración sea considerada una más de las posibles opciones laborales, no un imperativo extremo para la supervivencia de las y los trabajadores. Una política migratoria que despenalice la migración y resguarde los derechos humanos de los migrantes, sistemáticamente 
violados por el crimen organizado y las autoridades migratorias, es hoy imprescindible.

- Las respuestas políticas actuales frente al cambio climático tienden a centrarse en la atención de emergencias, no en revertir de fondo la degradación ambiental y la vulnerabilidad social. Conviene, entonces, tener una visión integral del riesgo para empezar a concentrarse en reducir el grado de vulnerabilidad social y ambiental. Los programas de adaptación al cambio climático y gestión ambiental deben articular acciones con los programas de reducción de riesgos, pero también con políticas económicas y sociales más amplias. La reducción de la vulnerabilidad social, el desempleo y la migración no pueden concebirse sin justicia económica y distributiva, sin mayor gobernabilidad y participación social. Los tratados internacionales en materia migratoria, laboral, de derechos humanos y de soberanía alimentaria, deben ser tomados en cuenta en el diseño de políticas y programas relativos al cambio climático.

\section{BIBLIOGRAFÍA}

- Aguilar, G. (2006). Los asentamientos humanos y el cambio climático en México. Un escenario futuro de vulnerabilidad regional. México: Instituto de Geografía de la UNAM.

- Adger, N. (2006). Vulnerability, Global Environmental Change 16: 268-281.

- Alscher, S. (2009). Environmental Change and Forced Migration Scenarios. México Case Study Report. EACHFOR. Obtenido desde www.each-for.eu

- Alscher, S. (2008). Environmental Change and Forced Migration Scenarios. General Overview-Latin America. EACH-FOR. Obtenido desde www.each-for.eul

- Ángeles, H. (2009) "Las múltiples dimensiones de la migración internacional en la frontera sur de México", en Serrano, J. \& Martínez, G. (coord) Una aproximación a las migraciones internacionales en la frontera sur de México. pp. 21-52. San Cristóbal Las Casas, Chiapas: REMISUR. Ecosur.

- Ángeles, H. \& Rojas, M. (2000) "Migración femenina internacional en la frontera sur de México". Papales de Población 23. pp. 127-151. México: UAEM.

- Ángeles, H. \& Rojas, M. (2009) "Temas pendientes sobre la migración internacional en la frontera sur de México", en Vargas P.; Giorguli S.; Castillo D.; García J. E.; RojasWiesner M. L.; Pederzini C.; Montes de Oca V.; Sandoval A.; Ordorica M. (Coords.). Foro Nacional Las políticas de Población en México. Programa Nacional de Población 2008-2012. Debates y propuestas. México: Consejo Nacional de Población. 
- Amnistía Internacional. (2010) Víctimas invisibles. Migrantes en movimiento en México. Madrid. Editorial Amnistía Internacional.

- Arellano, J. L. (1999). "El Manejo de Cuencas en Chiapas: Una estrategia para el Desarrollo Regional Sustentable", en: Medina-Mendoza Raúl. (Ed.). Memorias Simposio 4 Manejo Integral de Cuencas Hidrológicas. IX Congreso Nacional de Irrigación. pp. 29-48. Sinaloa: Asociación Nacional de Especialistas en Irrigación, A. C.

- Birkmann, J. (2009). Addressing the Challenge: Recommendations and Quality Criteria for Linking Disaster Risk Reduction and Adaptation to Climate Change. In: Birkmann; Joern; Tetzlaff; Gerd; Zentel; Karl-Otto (eds.) Bonn: DKKV Publication Series 38.

- Brown, O. (2008) Migración y cambio climático. Ginebra: Organización Internacional para las Migraciones.

- Calva, J.L. (2004). La reforma estructural de la agricultura y la economía en México: resultados y alternativas a nueve años del TLCAN, en: Schwentesius, R.; Gómez, M. A.; J. Calva \& L. Hernández. ¿El campo aguanta más? pp. 3- 21. México: CIESTAAM-UACh.

- CARE (2009). Climate Vulnerability and Capacity Analysis Handbook. CARE International. Obtenido desde: www.careclimatechange.org/cvca.

- CARE-UNU (2009). In Search of Shelter. Mapping the Effects of Climate Change on Human Migration and Displacement. CARE International, UN University, Columbia University. UN Refugee Agency (UNHCR).

- Castro, G. (2005). Las remesas de los migrantes. La migración en Chiapas. CIEPAC Boletín 480. Obtenido desde: www.ciepac.org/boletines/chiapasaldia.php

- CONAPO [Consejo Nacional de Población] (2007), México en cifras, Series sobre Migración. Obtenido desde: www.conapo.gob.mx

- CONEVAL. [Consejo Nacional de Evaluación de la Política de Desarrollo Social] (2012). Informe de Evaluación de la Política de Desarrollo Social 2011. Obtenido el 26 de agosto de 2012. Desde: www.coneval.gob.mx

- Couldrey, M. \& M. Herson. (2008) Cambio climático y desplazamiento. Revista Migraciones Forzadas No. 31. España: Centro de Estudios sobre Refugiados. Universidad de Alicante.

- Ellis, F. (2000). Rural Livelihoods and Diversity in Developing Countries. New York: Oxford University Press.

- EACH-FOR, (2009). Environmental Change and Forced Migration Scenarios Synthesis Report. Obtenido desde: www.each-for.eu/documents/EACHFOR_Synthesis_Report_090515.pdf

- Enciso, A. (2012). Sagarpa seguirá proceso de autorizaciones para cultivar maíz transgénico: Mayorga. La Jornada. p. 31. México. 
- Fetzek, S. (2009). Impactos relacionados con el clima en la seguridad Nacional de México y Centroamérica. Primer Informe. Instituto Real de Servicios Unidos-FUNDAECO. Gran Bretaña.

- Grupo Diálogo Rural (2011). Reunión de expertos para generar propuestas de políticas públicas para el campo. México. Obtenido el 04 de agosto de 2011. Desde: www.rimisp.org/proyectos/noticias_proy.php?id_proyecto $=262 \&$ id $=1196$.

- Ibarrarán, E. \& M. Rodríguez. (2007). Estudio sobre Economía del Cambio Climático en México. Instituto Nacional de Ecología y Universidad Iberoamericana Puebla.

- IOM, (2008) [International Organization for Migration], Migration and Climate Change, IOM Migration Research Series No. 31

- IOM, (2007) [International Organization for Migration], "Mexico: Facts and Figures". Desde: www.iom.int/jahia/Jahia/pid/484

- Intergovernmental Panel on Climate Change (2001). Climate Change: Impacts, Adaptation, and Vulnerability. Contribution of Working Group II to the Third Assessment. Cambridge University Press.

- Instituto Nacional de Ecología-SEMARNAT. (2006). Tercera Comunicación Nacional ante la Convención Marco de las Naciones Unidas sobre el Cambio Climático. México. INE; SEMARNAT; PNUD; EPA. Global Environment Facility.

- INEGI. (2005). Conteo Nacional de Población y Vivienda. Aguascalientes, México.

- INEGI. (2008). Censo Agropecuario 2007, IX Censo Ejidal. Aguascalientes. México.

- INEGI. (2009). Resultados de la Encuesta Nacional de Ocupación y Empleo en el Primer Trimestre de 2009 del Estado de Chiapas. Comunicado 143/09. Tuxtla Gutiérrez, Chiapas.

- INEG. (2010). Censo de Población y Vivienda 2010. México.

- Instituto Nacional de Migración. (2009) Encuesta sobre migración en la frontera Guatemala-México.

- Jungehülsing, J. (2010). Las que se van, las que se quedan: reacciones frente al cambio climático. Un estudio de caso sobre migración y género en Chiapas. Heinrich Böll Stiftung, Oficina México, Centroamérica y el Caribe. México.

- Jungehülsingj, J. (2011). Relaciones de género y vulnerabilidad de mujeres frente al cambio climático. Heinrich Böll Stiftung, Oficina México, Centroamérica y el Caribe.

- Kartiki, K. (2011) Climate change and migration: a case study from rural Bangladesh. Gender \& Development. 19 (1): 23-38. 
- Landa, R.; Magaña, V. \& Neri, C. (2008) Agua y clima: elementos para la adaptación al cambio climático. México: SEMARNAT.

- Lavell, A.; Oppenheimer, M.; Diop C.; Hess, J.; Lempert, R.; Li, J.; Muir-Wood, R. \& S. Myeong. (2012). Climate change: new dimensions in disaster risk, exposure, vulnerability, and resilience. In: Managing the Risks of Extreme Events and Disasters to Advance Climate Change Adaptation. Field, C.B.; Barros, V.; Stocker T.F.; Qin, D.; Dokken, D.J.; Ebi, K.L.; Mastrandrea, M.D.; Mach, K.J.; Plattner, G.K.; Allen, S.K.; Tignor M. \& Midgley P.M. (Eds.). A Special Report of Working Groups I and II of the Intergovernmental Panel on Climate Change (IPCC). Cambridge University Press, Cambridge, UK, and New York. USA, pp. 25-64.

- Magaña, V. \& Méndez, J. (2010) Escenarios de cambio climático para el estado de Chiapas. Informe elaborado para el Programa de Acción ante el Cambio Climático del Estado de Chiapas. Tuxtla Gutiérrez, Chiapas. Conservación Internacional México, A.C.

- Olivera, M. \& Sánchez, L. (2008). "Género: ¿estructura estructurante de la migración?”, en Villafuerte, D. \& García, C. Migraciones en el sur de México y Centroamérica. pp. 247-274. Porrua. UNICACH.

- Rojas, M. (2007) "Mujeres y migración en la frontera sur de México", Amérique Latine Histoire et Mémoire. Les Cahiers ALHIM, 14, Obtenido el 10 mayo 2010. Desde http://alhim.revues.org/index2252.html.

- Ruiz, L. (2011). Capacidades locales para la adaptación a la variabilidad climática en la cuenca del río Huehuetán, Chiapas. Informe de Investigación presentado al Fondo Mink’a de Chorlaví. Santiago, Chile.

- Saldaña, S. \& Sandberg, K. (2009). Impact of climaterelated disasters on human migration in Mexico: a spatial model. Climatic Change.

- Sánchez-Cohen, I.; Díaz-Padilla, G.; Ojeda, W.; Chebhouni, G.; Orona, I.; Villanueva, J.; González, J. \& González, G. (2008). Variabilidad climática en México: Algunos impactos hidrológicos, sociales y económicos. Ingeniería Hidráulica en México. Vol. XXIII. Núm. 4. 5-24.

- SAPRIN, (2002). Las políticas de ajuste estructural en las raíces de la crisis económica y la pobreza. Una evaluación participativa multi-nacional del ajuste estructural. Red Internacional de la Sociedad Civil para la Revisión Participativa de las Políticas de Ajuste Estructural. Washington, DC. Desde www.saprin.org

- Serrano, J. \& Tuñón, E. (2009) "Áreas de intensa emigración internacional en Tabasco: nuevos datos cuantitativos y cualitativos", en Serrano, J. y Martínez, G. (coord) Una aproximación a las migraciones internacionales en la frontera sur de México. pp. 99-122. San Cristóbal Las Casas, Chiapas: REMISUR, Ecosur. 
- Secretaría de Desarrollo Social, (2005). Boletín informativo 1. Delegación Chiapas. Tuxtla Gutiérrez.

- Valladares R.; Aguirre, R.; Duch, G.; Arias, M., Valladares, A. y Horanszky, R. (1999) El caos en Chiapas. Los siniestros de septiembre de 1998. SEMARNAP.

- Villafuerte, D. \& García, M. (2006) "Crisis rural y migraciones en Chiapas", en Migración y Desarrollo. Obtenido el 10 de marzo de 2010. Desde http://redalyc.uaemex.mx/redalyc/src/inicio/ArtPdfRed.jsp ?iCve $=66000604$.

- Villafuerte, D. \& García, M. (2007) "Veinte años de neoliberalismo en el campo chiapaneco". pp. 139-167. Anuario CESMECA 2006. Tuxtla Gutiérrez, Chiapas: UNICACH.

- Villafuerte, D. (2008) "Migración y desarrollo en el área del Plan Puebla- Panamá", en Villafuerte, D. \& García, C. Migraciones en el sur de México y Centroamérica. pp. 171219. México: Porrúa/UNICACH.

1. Dra. Laura Elena Ruiz Meza. Universidad de Ciencias y Artes de Chiapas. México laura.r.meza@gmail.com

2. La temporada de huracanes del año 2005 fue la más activa jamás registrada desde 1950 (INE-SEMARNAT, 2006). En México, los ciclos de fenómeno denominado $\mathrm{El}$ Niño/Oscilación del Sur (ENSO) es responsable de gran parte de la variabilidad climática interanual, fenómeno presente con mayor intensidad a partir de la década 1980 en comparación con períodos anteriores (Magaña \& Méndez, 2010).

3. Entre 1990 y 2002 la población mexicana indocumentada en Estados Unidos aumentó a 300 mil por año en promedio. Entre 2000 y 2005, alrededor de 400 mil mexicanos emigraron cada año. (Alscher, 2008).

4. Más del $95 \%$ del gasto en programas sociales está destinado a los pobres, pero sólo el $8 \%$ de los programas de fomento a la agricultura está dirigido a ese segmento. El incremento del presupuesto rural desde el 2001 no ha tenido un impacto positivo sobre el empleo rural, debido a que, en gran parte, la orientación de los subsidios favorece a los productores más adinerados, agudizando la desigualdad en las zonas rurales: $10 \%$ de los agricultores grandes concentra el $80 \%$ de los subsidios al campo (Grupo Diálogo Rural, 2011).

5. En sentido territorial, la frontera sur de México es una región constituida por cuatro entidades o provincias: Chiapas, Tabasco, Campeche y Quintana Roo, que limitan geográficamente con Guatemala y Belice.

6. Otras reformas estructurales fueron los cambios a la legislación agraria y de aguas que liberalizaron los 
derechos sobre la tierra y el agua para facilitar su privatización, afectando a los campesinos y beneficiando a las zonas de agricultura comercial destinadas a la exportación, las cuales han contado con el apoyo del Estado

7. Según criterios oficiales, el sector de la población que gana hasta dos salarios mínimos es considerado pobre

8. No se dispone de estadísticas exactas sobre el número de migrantes que entran en México. Según la IOM (2007) alrededor de 215 mil centroamericanos fueron detenidos y deportados por las autoridades mexicanas en 2004. El Instituto Nacional de Migración (INM) indica que el número de migrantes detenidos y devueltos a su país de origen fueron 64,061 centroamericanos en 2009, de los cuales 60,383 procedían de El Salvador, Guatemala, Honduras y Nicaragua. Del total 60,143 fueron repatriados voluntariamente o expulsados, a 2,846 se les permitió regularizar su condición de migrantes, y a 87 solicitantes de asilo se les otorgó la condición de refugiados. Una quinta parte eran mujeres y niñas (Amnistía Internacional, 2010).

9. Un estudio realizado en la Región del Soconusco, en Chiapas muestra que las familias campesinas subsisten mayoritariamente de ingresos no agrícolas: las transferencias proporcionadas por los programas públicos, el trabajo asalariado y las remesas aportaron el $67.6 \%$ de los ingresos familiares en 2009 (Ruiz, 2011). 